\title{
Le label NPO pour l'excellence du management
}

Christoph Gitz

Secrétaire général SSPP

Correspondance:

SSPP/FMPP

Secrétariat

Postgasse 17

CH-3000 Berne 8

ines.frutig[at]psychiatrie.ch
A l'occasion de la dernière assemblée des délégués, qui s'est tenue fin octobre, les responsables de la Société Suisse de Psychiatrie et Psychothérapie SSPP se sont vus remettre le certificat du label NPO pour l'excellence du management, couronnement d'un processus de développement continuel de plusieurs années de leur société de discipline médicale.

Impossible d'avoir deux poids deux mesures: la SSPP doit autant se soucier de la qualité que ses membres dans leur quotidien professionnel. Selon ce principe et dans le contexte du changement à la présidence et à la tête du secrétariat, les responsables de la SSPP ont commencé, il y a plus de deux ans, à esquisser et à réunir systématiquement tous les processus et documents essentiels au fonctionnement de la société et à les rendre accessibles en ligne à tous les collaborateurs dans le but de sauvegarder le savoir.

Alors que les processus éprouvés doivent simplifier l'entrée en fonction des nouveaux collaborateurs et membres du comité, les archives de documents servent à faciliter l'accomplissement des tâches quo- été mis en évidence où se situent les besoins de rattrapage et quels projets doivent être approfondis.

Le label NPO a été créé spécialement pour des sociétés/associations, de la SQS (Association Suisse pour Systèmes de Qualité et de Management) et du VMI (Institut pour le management des associations et autres organisations à but non lucratif). Le label, qui va plus loin que la certification ISO, est décerné aux associations et organisations à but non lucratif qui ont, de manière déterminée et avérée, organisé et implanté leur direction conformément aux connaissances disponibles à l'heure actuelle en matière de gestion. A l'aide d'une liste de points à vérifier spécifique, le système de gestion de l'organisation est évalué par des experts formés à cette tâche. Ceux-ci soumettent la demande de décernement du label, qui est officiellement remis par un comité paritaire composé de représentants du VMI et de la SQS.

Lors de l'assemblée des délégués du 29 octobre dernier, pour ainsi dire en même temps que le passage du témoin de la présidence, le président sortant de la

\section{«La SSPP doit autant se soucier de la qualité que ses membres dans leur quotidien professionnel.»}

tidiennes grâce à des modèles et des exemples adéquats. En passant par Internet, les responsables peuvent en tout temps accéder, dans le cadre de groupes d'utilisateurs définis tels que des commissions ou des groupes de travail ou de projet, aux processus qui les intéressent et aux documents qui y sont liés. Il est clair que les plus grandes améliorations au niveau du travail des collaborateurs auront lieu au secrétariat. Au cours du processus de certification, il a
SSPP Hans Kurt et le nouveau président Pierre Vallon ont reçu des mains du Lead-Assessor de la SQS, Roland Zürcher, le label NPO décerné sur la base de l'évaluation de certification du 10 et 11 août. A l'occasion de brèves évaluations de suivi annuelles, des mesures d'optimisation mineures sont définies et mises en œuvre dans la perspective d'un processus d'amélioration permanent. La certification est renouvelée tous les trois ans. 